RELATO DE CASO

\title{
Ressecção de aneurisma venoso em veia jugular externa direita
}

\author{
Resection of right external jugular vein aneurysm \\ Eduardo Pereira Savi', Fernando Wagner², Reginaldo Boppré2, Felipe Caetano Mamprim², Alberto Boppré2
}

\begin{abstract}
Resumo
O aneurisma venoso é uma anomalia rara, cujo diagnóstico pode ser realizado a partir de exames físicos e complementares. Sua raridade justifica a necessidade de investigação e de publicações de estudos de caso, objetivo maior deste estudo. Relata-se aqui o caso de uma paciente que apresentava um abaulamento cervical anterior assintomático, progressivo e com seis meses de evolução. A paciente foi submetida à cervicotomia anterior, sob anestesia geral, com ressecção do segmento venoso acometido e ligadura da veia jugular externa. Realizado o estudo, verificou-se que aneurismas venosos podem causar tromboflebite, embolia pulmonar ou rotura. Cirurgia profilática, quando oferece baixo risco, é cuidadosamente recomendada para pacientes com aneurismas abdominais e altamente recomendada para aneurismas do sistema venoso profundo dos membros inferiores. Outros aneurismas venosos devem ser tratados cirurgicamente quando sintomáticos, desfigurantes ou se apresentarem aumento progressivo.
\end{abstract}

Palavras-chave: Aneurisma; Esvaziamento cervical; Ligadura.

\begin{abstract}
Venous aneurysms are a rare abnormality, usually found in physical or complementary exams. We report a case of a 43-year old female with an asymptomatic and progressive enlarging mass in the neck. She had no history of trauma or cervical puncture. Vascular ultrasound showed a right jugular veins aneurysm with 1,81 x 1,62 cm of diameter. She was undergone resection and ligation of right external jugular vein, under general anesthesia. Venous aneurysm can cause thrombophlebitis, pulmonary embolism or spontaneous rupture. Prophylactic surgery is cautiously recommended for low-risk patients with venous aneurysms of the abdomen and strongly recommended for most patients with lower extremity deep venous aneurysms. Other venous aneurysms should be excised only if they are symptomatic, enlarging, or disfiguring
\end{abstract}

Keywords: Aneurysm; Neck dissection; Ligation.

Introdução

Aneurismas venosos são raros, acometem pessoas de qualquer sexo e idade, e podem afetar qualquer veia ${ }^{1}$. A etiologia do aneurisma venoso permanece incerta, embora várias hipóteses tenham sido elaboradas ${ }^{2,3}$. Por sua raridade, os aneurismas venosos são pouco descritos; há relatos esporádicos na literatura mundial. Em quatro grandes revisões, foram relatados 147 casos por Calligaro et al. $^{4}, 311$ por Schild et al. ${ }^{5}, 39$ por Gillespie et al. ${ }^{6}$.

Embora a tomografia computadorizada, a flebografia e a ressonância magnética auxiliem o diagnóstico preciso, o padrão-ouro para método diagnóstico de aneurisma venoso é a ultrassonografia com dúplex cor fluxo de imagens $s^{7-9}$.

\section{Relato de caso}

Paciente do sexo feminino, com 43 anos de idade e uma história de abaulamento (Figura 1) na região cervical anterior, assintomático progressivo e com seis meses de evolução (Figura 2). A paciente apresentava asma brônquica como comorbidade.

$\mathrm{O}$ exame de ecodoppler venoso mostrou imagem anecoica, compressível e com 1,81 x 1,62 cm de diâmetro, com fluxo venoso fásico em seu interior, compatível com aneurisma de veia jugular externa.

O tratamento cirúrgico foi sugerido, para o qual foram solicitados exames pré-operatórios. Esses exames apresentaram hemograma normal, com eritrócitos de 4,8 milhões; hemoglobina de 13,3 g/dL; hematócrito de $40 \%$;

Trabalho realizado no Hospital Nossa Senhora da Conceição (HNSC), Tubarão (SC), Brasil.

${ }^{1}$ Acadêmico do Curso de Medicina da Universidade do Sul de Santa Catarina (Unisul), Tubarão (SC), Brasil.

${ }^{2}$ Cirurgião Vascular do Hospital Nossa Senhora da Conceição (HNSC), Tubarão (SC), Brasil.

Submetido em: 14.01.2010 Aceito em: 28.07.2010

J Vasc Bras. 2010;9(4):245-248. 


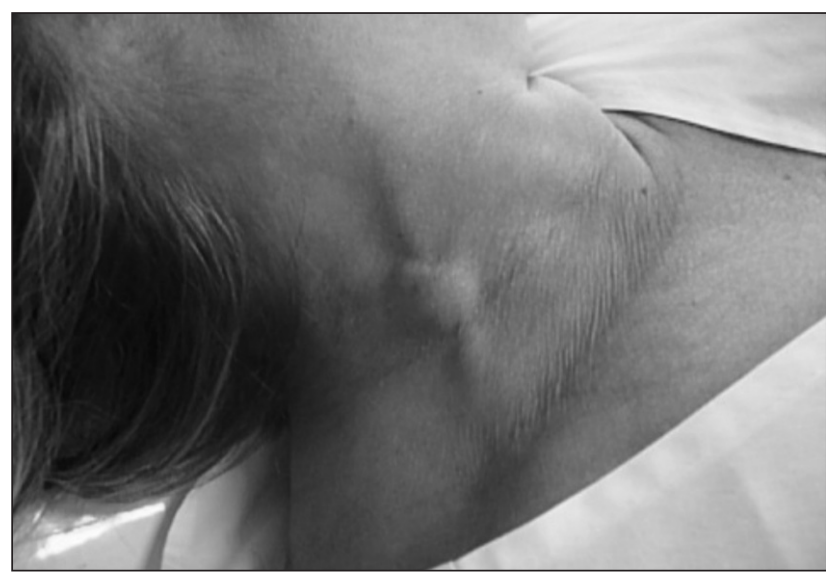

Fonte: própria, 2009.

Figura 1 - Massa subcutânea; pescoço, lado direito.

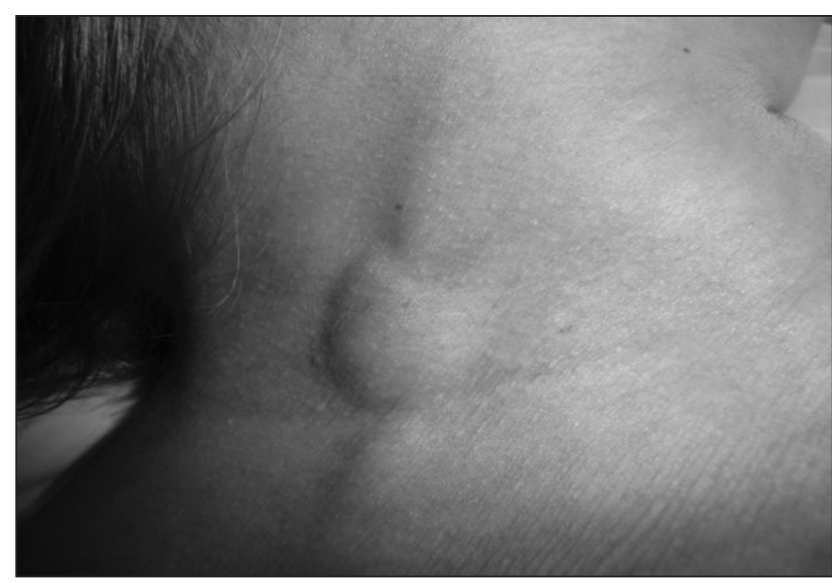

Fonte: própria, 2009

Figura 2 - Massa subcutânea; pescoço, lado direito.

hemácias normocíticas e normocrômicas; colesterol total de $160 \mathrm{mg} / \mathrm{dL}$; triglicerídeos de $58 \mathrm{mg} / \mathrm{dL}$; creatinina de 0,57 mg/dL; glicose de $92 \mathrm{mg} / \mathrm{dL}$; plaquetas 243.000; Tap 14 segundos; tempo de sangramento 1'05" minutos; KPTT 30 segundos.

A paciente foi submetida à cervicotomia anterior (Figuras 3 e 4), sob anestesia geral, e à ressecção do aneurisma (Figura 5) com ligadura da veia jugular externa (Figura 6). A paciente obteve alta no segundo dia pós-operatório e a evolução foi favorável (Figura 7 e 8).

\section{Tratamento}

O tratamento, dependendo da localização anatômica do aneurisma, pode ser conservador, com a simples observação de sua evolução, ou cirúrgico ${ }^{3}$. Uma das sugestões de tratamento de um aneurisma venoso é a ligadura das veias proximais e distais ${ }^{10}$.

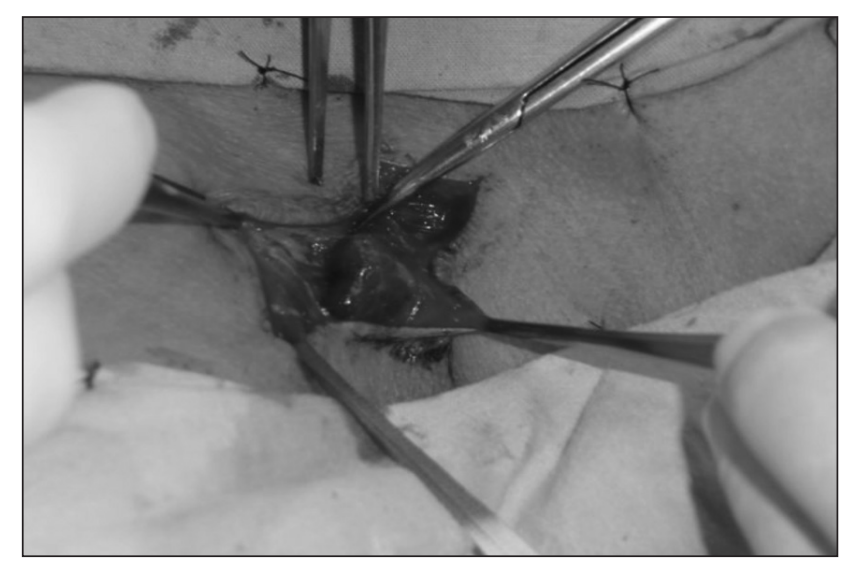

Fonte: própria, 2009

Figura 3 - Dissecção de aneurisma em veia jugular.

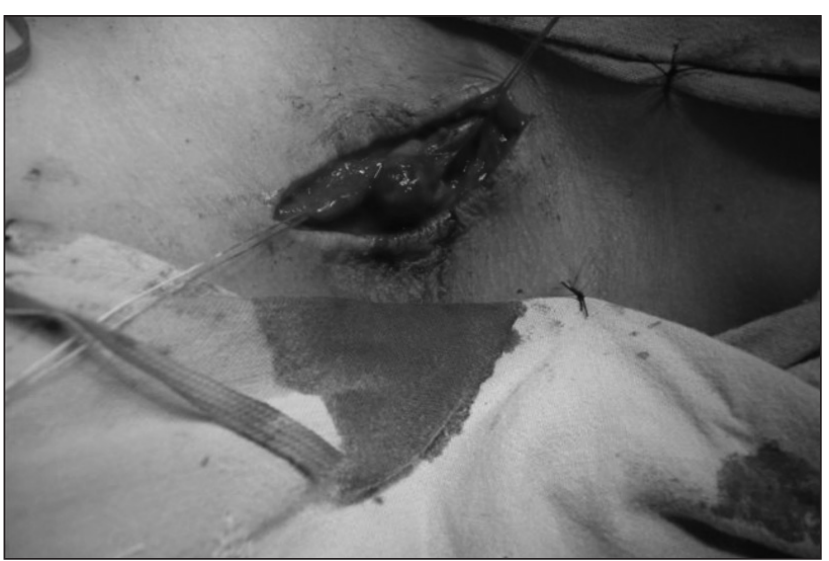

Fonte: própria, 2009

Figura 4 - Dissecção de aneurisma em veia jugular.

\section{Discussão}

A primeira descrição de aneurisma venoso foi feita por Osler, em 1913, em um caso de dilatação de veia axilar associado a aneurisma da artéria homônima ${ }^{11}$. Schild et al. ${ }^{5}$ relataram apenas 311 casos em toda literatura, de 1939 até 1992, sendo a maioria de veia jugular interna. Essa realidade reforça a necessidade de publicações de relatos de casos de aneurisma venoso, especialmente os de jugular externa.

Aneurismas venosos podem acometer neonatos, crianças, adolescentes, adultos e idosos e podem se desenvolver em qualquer parte do sistema vascular ${ }^{2,12-14}$. Os aneurismas venosos podem se apresentar como fusiformes ou saculares; essa distinção é importante para a estratégia cirúrgica ${ }^{3}$.

Os aneurismas de veia jugular externa podem ser diagnosticados por meio de exame físico que possibilita verificar um abaulamento do pescoço ou uma suave e compressível massa na região cervical. Em relação ao exame 


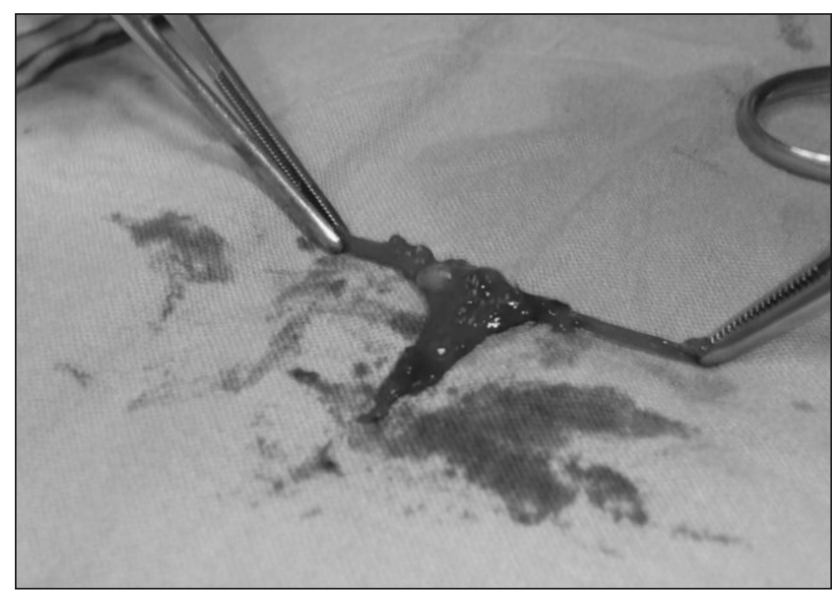

Fonte: própria, 2009.

Figura 5 - Aneurisma venoso seccionado.

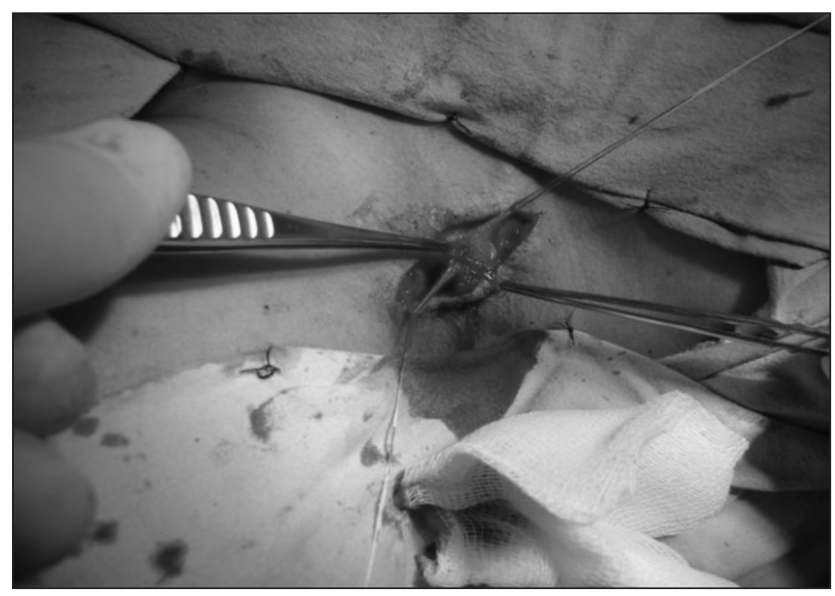

Fonte: própria, 2009.

Figura 6 - Ligamento do coto proximal e distal do aneurisma.

complementar por imagem, o ecodoppler oferece subsídios para um diagnóstico acurado ${ }^{15-17}$.

Embora os aneurismas venosos sejam raros, não devem ser menosprezados. Podem apresentar risco significativo de morbidade e mortalidade por ruptura e hemorragia, ou por trombo e eventual formação de embolia pulmonar $^{18}$.

Os aneurismas venosos são classificados em diferentes tipos. Um aneurisma venoso primário é definido como uma área de isolamento de dilatação venosa que se comunica com uma estrutura venosa por um único canal e não deve ter associação com comunicação arteriovenosa ou aneurisma. Já o aneurisma venoso secundário ou adquirido é geralmente encontrado em adultos e, mais frequentemente, associado ao traumatismo, à fístula arteriovenosa ou à alteração hemodinâmica venosa ${ }^{10}$.

Aneurisma congênito de veia é uma anomalia do desenvolvimento da parede venosa, que ocorre como

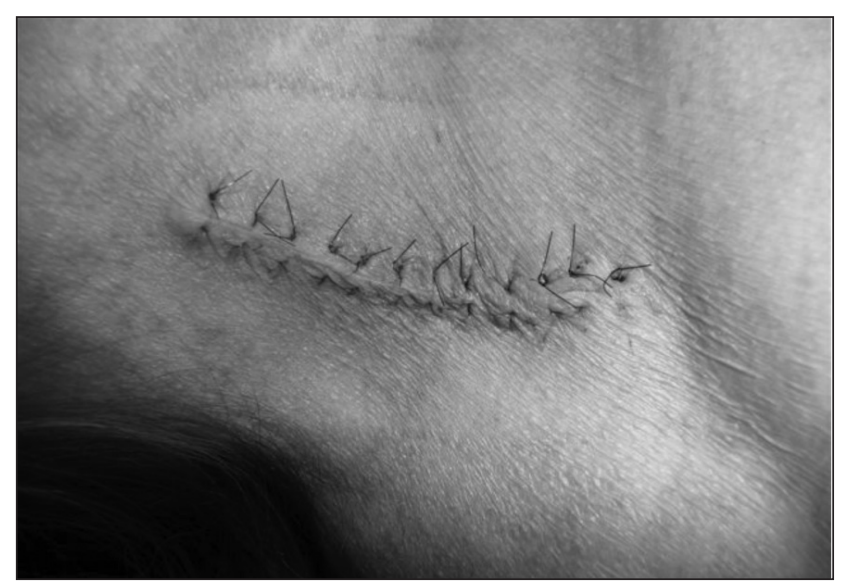

Fonte: própria, 2009.

Figura 7 - Cicatriz da cirurgia ; aneurisma venoso.

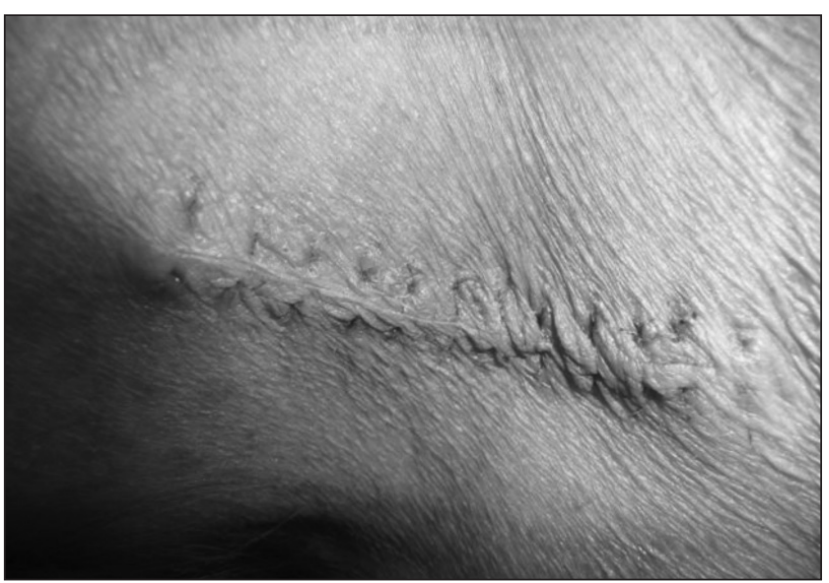

Fonte: própria, 2009.

Figura 8 - Cicatriz da cirurgia; aneurisma venoso.

consequência da hipoplasia do músculo liso, levando à deficiência elástica da parede venosa ${ }^{19}$. A etiologia do aneurisma foi descrita como degeneração da parede venosa, resultando em adelgaçamento da camada elástica e muscular da parede da veia. Outra maneira fisiopatológica seria um prolongado estresse mecânico da parede venosa ${ }^{8}$.

Ilijevski et al. ${ }^{20}$, em seu estudo anatomopatológico de aneurisma venoso, mostraram todas as camadas e a continuidade endotelial da veia normais, com proliferação de fibroblastos de mistas camadas musculares. São pontos de vista que merecem maior investigação.

\section{Conclusão}

Aneurismas de veia jugular externa são anomalias raras e aparecem como um suave e compressível abaulamento no pescoço ${ }^{21}$. Diferente dos aneurismas arteriais, 
exaustivamente estudados e relatados na literatura, os aneurismas venosos são achados raros na prática médica. Desse modo, sua ocorrência é sempre motivo de publicação ${ }^{22,23}$.

O diagnóstico clínico do aneurisma venoso é feito por intermédio de anamnese, de exame físico, e o diagnóstico definitivo é obtido por ecodoppler colorido ${ }^{20}$; flebografia, tomografia computadorizada e ressonância magnética também podem auxiliar o diagnóstico ${ }^{9,17}$.

Embora o aneurisma venoso seja considerado um caso relativamente simples, o tratamento cirúrgico é indicado na maioria dos casos, principalmente quando sintomáticos, desfigurantes ou que apresentem aumento progressivo.

\section{Referências}

1. Suchý T. [Venous aneurysms]. Rozhl Chir. 1995;74:284-6. [Article in Czech].

2. Faraj W, Selmo F, Hindi M, Haddad F, Khalil I. Cephalic vein aneurysm. Ann Vasc Surg. 2007;21:804-6.

3. Dourado OC, Miranda AG, Pinheiro Filho A, et al. Aneurisma venoso no pé: relato de casos e revisão da literatura. J Vasc Bras. 2006;5:313-6.

4. Calligaro KD, Ahmad S, Dandora R, et al. Venous aneurysms: surgical indications and review of the literature. Surgery. 1995; 117:1-6.

5. Schild H, Berg S, Weber W, et al. [The venous aneurysm]. Aktuelle Radiol. 1992;2:75-80. [Article in German].

6. Gillespie DL, Villavicencio JL, Gallagher C, et al. Presentation and management of venous aneurysms. J Vasc Surg. 1997;26:845-52.

7. Ahn JY, Chung YS, Lee BH, et al. J Stent-graft placement in a traumatic internal carotid-internal jugular fistula and pseudoaneurysm. Clin Neurosci. 2004;11:636-9.

8. Ilijevski NS, Radak S, Vucurević G, et al. Jugular vein aneurysm. Vascular. 2008;16:291-4.

9. Verbeeck N, Hammer F, Goffette P, et al. Saccular aneurysm of the external jugular vein, an unusual cause of neck swelling. J Belge Radiol. 1997;80:63-4.

10. Majeski J. Surgical repair of primary saphenous vein aneurysm of the proximal leg after initial presentation as an inguinal hernia. Am Surg. 2002;68:999-1002.
11. Castle JM, Arous EJ. Femoral vein aneurysm: a case report and review of the literature. Cardiovasc Surg. 1995;3:317-9.

12. Kuss J), Karli A, Fischbach $M$, et al. Congenital carotid to jugular aneurysm. Arch Fr Pediatr. 1979;36:502-7. [Article in French].

13. Beale TJ, Smedley FH, Knee G. Thrombosis within an external jugular venous aneurysm. JR Coll Surg Edinb. 1996;41:181-2.

14. Gillespie DL, Villavicencio JL, Gallagher C, et al. Presentation and management of venous aneurysms. J Vasc Surg. 1997;26:845-52.

15. Hermans R. Phlebectasia of the external jugular vein: a case report. J Belge Radiol. 1991;74:221-2.

16. Dhillon MK, Leong YP. Singapore Jugular venous aneurysm - a rare cause of neck swelling. Med J. 1991;32:177-8.

17. Andreev A, Petkov D, Kavrakov T, et al. Jugular venous aneurysms: when and how to operate. Int Angiol. 1998;17:272-5.

18. Friedman SG, Krishnasastry KV, Doscher W, Deckoff SL. Primary venous aneurysms. Surgery. 1990;108:92-5.

19. Sitkovski NB, Danhin TI, Kisel NP, et al. Pathogenesis of congenital aneurysm of the internal jugular veins. AN Khirurgiia (Mosk). 1993;(8):22-5. [Article in Russian].

20. Ilijevski NS, Radak S, Novakovic B, et al. Images in vascular medicine. Jugular vein aneurysm--ultrasonographic evaluation. Vasc Med. 2006;11:51.

21. Porcellini $M$, Selvetella $L$, Bernardo $B$, et al. [Aneurysms of the external jugular vein]. Chir. 1996;17:238-41. [Article in Italian]

22. Sessa C, Nicolini P, Perrin M, et al. Management of symptomatic and asymptomatic popliteal venous aneurysms: a retrospective analysis of 25 patients and review of the literature. J Vasc Surg. 2000;32:902-12.

23. Wolosker N, Zerati AE, Nishinari K, et al. Aneurysm of superior mesenteric vein: case report with 5-year follow-up and review of the literature. J Vasc Surg. 2004;39:459-61.

Correspondência: Eduardo Pereira Savi

Unidade Hospitalar de Ensino da Universidade do Sul de Santa Catarina Avenida Marcolino Martins Cabral, s/n., anexo ao Hospital Nossa Senhora da Conceição CEP: 88701-900 - Tubarão (SC), Brasil E-mail: edu-savi@hotmail.com

Contribuições dos autores: Pesquisa e redação: EPS Relato de caso: FW, RB, AB Fotos: RB, $A B$ Texto em inglês: FC 